\title{
Offline Handwritten Hindi 'SWARs' Recognition Using A Novel Wave Based Feature Extraction Method
}

\author{
Ajay Indian', Karamjit Bhatia ${ }^{2}$ \\ ${ }^{1}$ Associate Professor, Invertis University, Bareilly \\ \& Research Scholar, Gurukula Kangri Vishwavidyalaya, Haridwar, India \\ ${ }^{2}$ Professor, Department of Computer Science \\ Gurukula Kangri Vishwavidyalaya, Haridwar, India
}

\begin{abstract}
Offline handwritten character recognition is a very challenging area of research as handwriting of two persons may bear resemblance whereas handwriting of an individual may vary at different times. The character recognition accuracy depends on the ways the features are extracted from the samples and utilized to formulate the feature vector. In this paper, a novel technique 'TARANG' for feature extraction is proposed to recognize offline handwritten Hindi 'SWARs' (vowels). This technique for extracting features from an image is inspired by the natural movement of wave in a medium. A feature vector obtained by using proposed technique is used for the training of Backpropagation Neural Network and recognition rate as high as $96.2 \%$ is achieved.
\end{abstract}

Keywords- Offline Handwritten Character Recognition, Feature Extraction, Global Features, Local Features, Back-propagation Neural Network.

\section{Introduction}

Pattern recognition (PR) broadly refers to an area of machine learning and intelligence. The problem of pattern recognition has several aspects and this problem has been addressed in many ways. A widely addressed and an important aspect of the problem is the pattern classification. The pattern classification is a problem in which a machine, when served different input stimuli, performs the differentiation according to the features present in the inputs in some meaningful categorization.

The character recognition comes under the umbrella of pattern recognition [1]. The problem of character recognition is categorized as offline character recognition and online character recognition. In offline character recognition, typed/ handwritten characters are scanned and converted into binary or gray scale image. Then, feature extraction and recognition process is carried over the binary image. Offline character recognition is complex tasks as no timing information about character strokes is available. Therefore, offline character recognition can be thought as a more challenging task than its online counterpart. In online character recognition, writing and recognition are done simultaneously. A user writes a character on any sensory area where sensors pick up the pen movements and then on the basis of those pen movements characters are recognized. As timing information is available, online character recognition is much easier than offline character recognition [2][3].

The process of character recognition involves several phases which include collecting samples from different people, applying pre-processing on the collected samples to make them ready to extract features, executing an effective and efficient algorithm to extract features for feature vector creation, and applying a classification strategy to assign particular character to a specific class. A number of feature extraction techniques have been proposed by the researchers to mine the specific features. Feature extraction methods for handwritten character recognition are based on either structural features or statistical features. For classification and training purposes several algorithms like BPNN, KNN, SVM, genetic algorithms etc. have been proposed [4] [5]. The various steps involved in a character recognition system are depicted in Fig. 1. In context of present study, character recognition means recognition of offline handwritten Hindi 'SWARs' (vowels) showed in the Fig. 2.

The rest of the paper is arranged as: Literature survey related to offline handwritten Hindi character recognition is presented in Section II. Section III gives motivation and objective of the research work, Section IV describes the proposed approach. Section $\mathrm{V}$ is devoted to implementation of the proposed approach, Section VI is devoted to result discussion and finally the conclusion of this paper is given in section VII. 


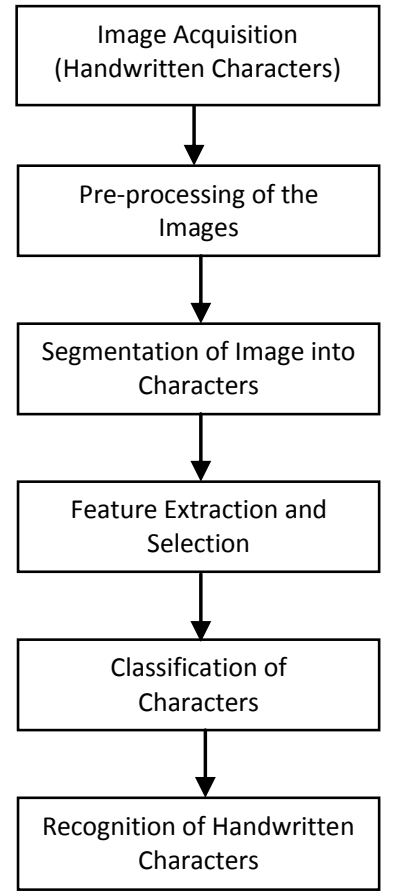

Figure 1: Steps in Handwritten Character Recognition System

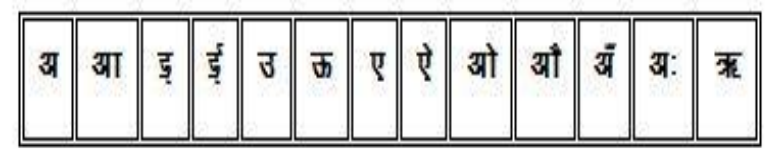

Figure 2: 'SWARs' (vowels) in Hindi Script

\section{A Survey on Offline Handwritten Hindi Character Recognition}

The task of offline handwritten character recognition has been attempted by many researchers with different approaches. A lot of work has been reported for recognition of characters written in different languages like English, Hindi, Japanese, Kannad, Bangla, Chinese etc. Different techniques such as ANNs, Genetic Algorithms, Fuzzy logic, KNN, SVM etc. and various approaches like Clonal Selection Algorithm, Bacterial Foraging, Gradient features method, Hidden Markov Model etc. have been employed for the handwritten character recognition.

Utpal Garain et al. [6] explored the potentiality of a Clonal Selection Algorithm in pattern recognition. A retraining scheme for the Clonal Selection algorithm was formulated for recognition of handwritten numerals considering it a 10-class classification problem. Experimental results revealed $96 \%$ average recognition accuracy. Ranadhir Ghosh and Moumita Ghosh [7] proposed a technique, a hybrid evolutionary method, which used a combination of genetic algorithm and matrix based solution methods.

Hanmandlu et al. [8] presented the recognition of handwritten Hindi characters based on the customized exponential membership function fitted to the fuzzy sets developed from features containing normalized distances obtained using the Box approach. A database of 4750 samples was used for the study and authors claimed $90.65 \%$ overall recognition rate. M. Hanmandlu et al. [9] also worked on the recognition of handwritten Hindi numerals using modified exponential membership function, fitted to the fuzzy sets, derived from features consisting of normalized distances obtained using the Box approach. The optimization approach employed was the foraging model of E.coli bacteria. Overall recognition rate claimed by the authors was $96 \%$.

U. Pal et. al. [10] presented a comparative study of Devanagari handwritten character recognition using twelve different classifiers and four set of features. Feature sets used in the classifiers calculated the curvature and gradient information acquired from binary as well as gray-scale images. Mukherji and Rege [11] proposed a shape based technique for recognition of isolated handwritten Devanagari characters. The position in the image frame was based on fuzzy classification. The average accuracy of recognition of the developed system was claimed to be $86.4 \%$.

Sandhya Arora et al. [12] applied weighted majority voting method for a combination of classification decision obtained from three Multi-Layer Perceptron (MLP) based classifiers using three different feature sets. Simulation study, with a dataset of 4900 samples, revealed an overall recognition rate of 92.16\%. Sheetal Dabra et al. [13] discussed the recognition of Similar Shaped Handwritten Hindi Characters (SSHHC) using four ML algorithms namely Bayesian Network, RBFN, MLP and C4.5. The performance of four algorithms was analyzed and compared. It was found that MLP gave better performance with an increase in the number of samples of the same target pair in the training dataset.

Gunjan Singh et al. [14] presented a system for handwritten Hindi character recognition. Feature sets were developed by counting the number of character pixels and background pixels of the normalized character image. Experimental results showed that back-propagation network yielded recognition accuracy of $93 \%$. Rakesh Rathi et al. [15] proposed a feature extraction method based on recursive sub division technique and implemented on Devanagari vowels with KNN classifier. During the recognition process using KNN classifier 88 vowels were wrongly identified out of 2281vowels thereby producing $96.14 \%$ recognition rate.

Sonika Dogra et al. [16] presented a recognition system using Support Vector Machine as a classifier and diagonal feature extraction approach was used to extract features. The system 
gave $93.06 \%$ accuracy in Hindi handwritten character recognition. Gaurav Y. Tawde [17] presented a method for recognition of isolated offline handwritten Devanagari numerals using wavelets and neural network classifier. The feed forward back propagation algorithm was used for classification of the input numeral. The recognition accuracy obtained by this method was in the range $60 \%-70 \%$. Kulkarni Sadanand A. et al. [18] described the efficiency of Zernike complex moments and Zernike moments with different zoning patterns for offline recognition of handwritten characters. The work produced $94.78 \%$ accuracy in recognition by using Zernike complex moments and $94.92 \%$ by using Zernike moments.

\section{Motivation and Objective}

Literature survey reveal that various approaches have been proposed for offline handwritten character recognition but the achieved recognition rate of handwritten characters is not satisfactory, specifically in case of handwritten Hindi character recognition. It is further identified that recognition of typed characters is easier than handwritten characters. Further, due to unavailability of dynamic information, the development of an efficient and reliable offline handwritten character recognition system is supposed to be a tough task as compared to the development of its online counterpart. The complexity of Hindi script and bi-lingual characteristic of Hindi script makes it more difficult to achieve the higher recognition rate for Offline handwritten Hindi characters. Thus, in the context of Offline handwritten Hindi character recognition, there is still a great scope of work to propose techniques for achieving recognition rate close to $100 \%$. Hence, there is a need for efficient techniques for offline handwritten Hindi character recognition.

The prime aim of the proposed work is to propose an efficient approach for feature extraction to enhance the accuracy of offline handwritten character recognition of Hindi 'SWARs' (vowels).

\section{Proposed Approach}

Proposed approach for feature extraction is inspired from the wave created when a stone is thrown in a pond. Wave is created at the point of contact of stone and water (say, in the centre) and it travels towards the boundary (outward) of the pond in spherical shape. At the starting, wave is very strong but as it travels towards the outward it becomes weaker and weaker and at last, it vanishes. The phenomenon is employed in feature extraction from an image with intent that the middle portion of the image is more significant to extract the features as compared to the frontier (outer) portions.

The proposed method of feature extraction, called 'TARANG', starts with calculating the features of an image by dividing it into different wave zones starting from the centre of the image and gradually increasing the number of pixels in order to reach the boundary (outward) of the image. Before extracting the features using the proposed approach, images are passed through the FFT in order get the image with less noise and image with special frequency domain, such that special features may be extracted. The proposed approach is described next:

\section{Image acquisition Phase}

Step 1- Samples of handwritten Hindi SWARs from different persons of varying age groups are collected on the white paper sheet of A4 size.

Step 2- All paper sheets with handwritten Hindi SWARs samples are scanned and images are produced in digital form.

\section{Pre-processing Phase}

Step 3- All images are converted into black and white images using global threshold method.

Step 4- The images obtained in step 3, are passed through the FFT.

Step 5- The FFT images are then passed through a 2-D Filter. Step 6 - Images produced are then passed through the inverse FFT to get the images with less noise and with in special frequency domain.

\section{Segmentation Phase}

Step 7- Segmentation of all images produced in step 6 is done using bounding box method in order to isolate each character from the samples image.

Step 8- Each isolated image (character) is resized into a binary image of $40 \times 40$ pixels.

\section{Feature Extraction and Selection Phase}

Step 9-Features of each sample image are calculated as below:

i) Global Wave Feature Extraction

a. Each sample image is divided into 10 wave zones as shown in Fig. 3.

b. The average intensity $A_{i}(i=1,2,3 \ldots, 10)$ of each wave zone is calculated and a feature vector $A=\left\{A_{1}\right.$, $\left.\mathrm{A}_{2}, \mathrm{~A}_{3} \ldots, \mathrm{A}_{10}\right\}$ is obtained.

ii) Local Wave Feature Extraction

a. Each sample image is divided into four quadrants of size $20 \times 20$ pixels and each quadrant is divided into 6 wave zones as shown in Fig. 4.

b. The average intensity $B_{i}(i=1,2,3 \ldots, 24)$ of each wave zone is calculated and a feature vector $B=\left\{B_{1}\right.$, $\left.\mathrm{B}_{2}, \mathrm{~B}_{3} \ldots, \mathrm{B}_{24}\right\}$ is obtained.

iii) Local Feature Extraction without Wave Zones

a. Each sample image is divided into four quadrants of size $20 \times 20$ pixels as shown in Fig. 5.

b. The average intensity $\mathrm{C}_{\mathrm{i}}(\mathrm{i}=1,2 \ldots, 4)$ of each quadrant is calculated and a feature vector $\mathrm{C}=\left\{\mathrm{C}_{1}, \mathrm{C}_{2}\right.$, $\left.\mathrm{C}_{3}, \mathrm{C}_{4}\right\}$ is obtained. 


\section{Classification and Recognition Phase}

Step 11-Feed Forward Back-propagation Neural Network is trained.

Step 12- The trained neural network is used for classification and recognition.

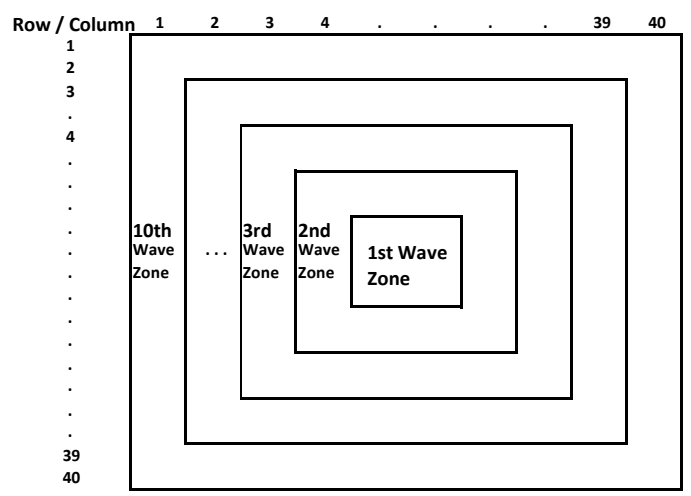

Figure 3: Division of an Image into 10 Wave Zones

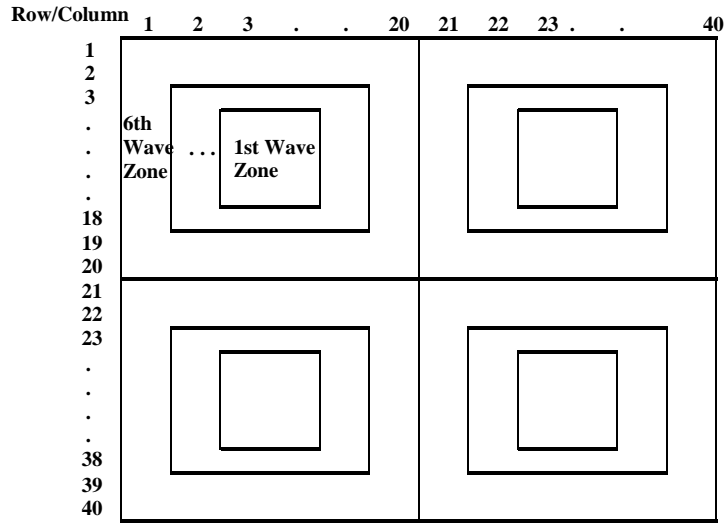

Figure 4: Division of Image into 4 Quadrants and Division of Each Quadrant into 6 Wave Zones

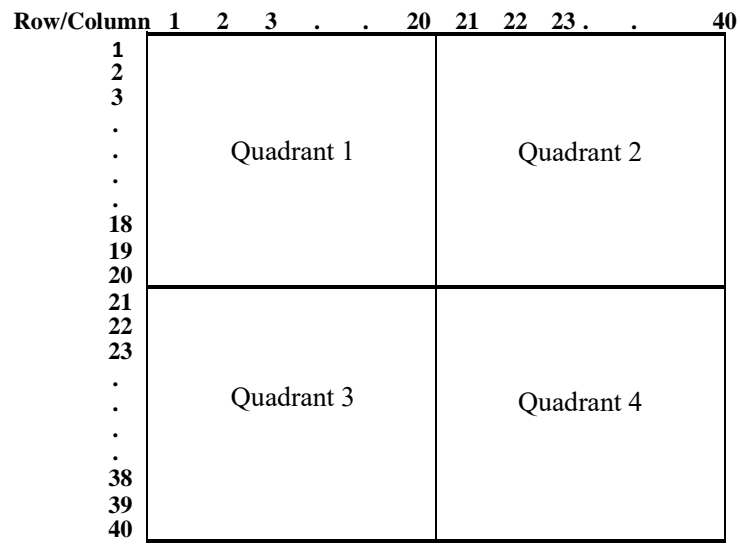

Figure 5: Division of Image into 4 Quadrants without wave zones

\section{Implementation}

Samples of all (13) Hindi vowels (SWARs) is collected on the white paper sheet of A4 size from different persons (150) of varying age group to create a database of 1950 samples. All the samples taken on the paper are converted to digital form by scanning the sheets through a flatbed scanner with an optical resolution of 2400 x 4800 dpi. Samples of handwritten Hindi SWARs collected from three different persons are shown in Table 1.

Table 1. Samples of Handwritten Vowels in Hindi Script Collected from Three Persons

\begin{tabular}{|c|c|c|c|}
\hline S. No. & Sample 1 & Sample 2 & Sample 3 \\
\hline 1 & अT & अ & 3 \\
\hline 2 & आ & जा & $3 \pi$ \\
\hline 3 & $\bar{s}$ & $\xi$ & $\widehat{\hat{S}}$ \\
\hline 4 & $\xi$ & $\xi$ & $\$$ \\
\hline 5 & $\overline{3}$ & 3 & \\
\hline 6 & $\overline{3}$ & 3 & 3 \\
\hline 7 & ए5 & Z & 7 \\
\hline 8 & $\vec{E}$ & ऐो & tr \\
\hline 9 & ओ & ओ & ओ \\
\hline 10 & औ & और & औ \\
\hline 11 & अ & अं & उं \\
\hline 12 & 310 & $\overline{3 !}$ & अ: \\
\hline 13 & $\overline{A L}$ & 便 & 㓞 \\
\hline
\end{tabular}

To study the efficacy of the created feature vectors in offline handwritten character recognition following four strategies are considered:

Strategy I- Consisted of global wave feature vector A of length 10 for training the network.

Strategy II- Consisted of local wave feature vector B of length 24 for training the network.

Strategy III- Consisted of combined wave feature vector $\mathrm{C}=\{\mathrm{A}\} \cup\{\mathrm{B}\}$ of length 34 for training the network.

Strategy IV- Consisted of combined feature vector $D=\{A\}$ $\mathrm{U}\{\mathrm{B}\} \mathrm{U}\{\mathrm{C}\}$ of length 38 for training the network. 
MATLAB 2013a is used to carry out the experiments using the sample set of vowels. Keeping in view the complexity of character recognition problem, neural network architecture with two hidden layers is adopted. For training phase, Backpropagation Neural Network (BPNN) with two hidden layers $(\mathrm{H} 1$ and $\mathrm{H} 2)$ is used. The output layer $(\mathrm{O})$ of neural network consisted of thirteen neurons for representing the thirteen different classes of Hindi vowels whereas the number of neurons in the input layer (I) depended upon the lengths of feature vectors. Table 2 depicts the features, feature length and the architecture of neural network for different strategies.

Table 2. Features, Feature length and Architecture of Neural Network for Different Strategies

\begin{tabular}{|c|c|c|c|}
\hline Strategy & Features & $\begin{array}{c}\text { Length of } \\
\text { Feature } \\
\text { Vector }\end{array}$ & $\begin{array}{c}\text { Neural Network } \\
\text { Architecture } \\
\text { (I-H1-H2-O) }\end{array}$ \\
\hline I & $\begin{array}{c}\text { Global wave } \\
\text { feature }\end{array}$ & 10 & $10-5-5-13$ \\
\hline II & $\begin{array}{c}\text { Local wave } \\
\text { feature }\end{array}$ & 24 & $24-12-12-13$ \\
\hline III & $\begin{array}{c}\text { Combined } \\
\text { wave feature }\end{array}$ & 34 & $34-24-13-13$ \\
\hline IV & $\begin{array}{c}\text { Combined } \\
\text { feature }\end{array}$ & 38 & $38-24-13-13$ \\
\hline
\end{tabular}

Once the neural network is trained to classify and recognize the new handwritten vowels (test samples) of Hindi script. Neural networks is trained for all the strategies I, II, III and IV. During the testing phase, 650 samples are selected randomly out of total 1950 samples.

\section{Experimental Results}

The performance of various methods in terms of training time (in second) and recognition rate (in \%) is presented in Table 3. It is obvious from Table 3 that global wave features (strategy I) and local wave features (strategy II) individually achieved lower recognition rate $73.2 \%$ and $84.5 \%$, respectively. But, when global wave features and local wave features are combined (strategy III) a higher recognition rate of $95.7 \%$ is achieved. Slightly better recognition rate of $96.2 \%$ is achieved in strategy IV when average intensity feature vector of four quadrants without wave zone is combined with feature vector of strategy III.

Table 3. Performance of Different Strategies

\begin{tabular}{|c|c|c|}
\hline Strategy & $\begin{array}{c}\text { Training Time } \\
\text { (in seconds) }\end{array}$ & $\begin{array}{c}\text { Recognition Rate } \\
\text { (in \%) }\end{array}$ \\
\hline I & 81 & 73.2 \\
\hline II & 107 & 84.5 \\
\hline III & 149 & 95.7 \\
\hline IV & 161 & 96.2 \\
\hline
\end{tabular}

Recognition rates (accuracies) of individual characters using Back-propagation Neural Network with four strategies are shown in Table 4. For strategy IV, more confusion was found in recognizing characters ${ }^{3}$, ए, औ and , , Comparatively less confusion was recorded in recognizing

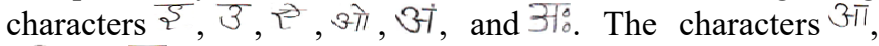
$\$$ and $\bar{\zeta}$ are recognised with $100 \%$ accuracy.

Table 4. Recognition Rate (in \%) of Individual Characters with Different Strategies

\begin{tabular}{|c|c|c|c|c|c|}
\hline $\begin{array}{c}\text { S. } \\
\text { No. }\end{array}$ & $\begin{array}{c}\text { Hindi } \\
\text { SWARs } \\
\text { (Vowels) }\end{array}$ & $\begin{array}{c}\text { Strategy } \\
\text { I }\end{array}$ & $\begin{array}{c}\text { Strategy } \\
\text { II }\end{array}$ & $\begin{array}{c}\text { Strategy } \\
\text { III }\end{array}$ & $\begin{array}{c}\text { Strategy } \\
\text { IV }\end{array}$ \\
\hline 1 & उT & 70 & 90 & 98 & 90 \\
\hline 2 & आ & 82 & 84 & 96 & 100 \\
\hline 3 & S & 60 & 76 & 98 & 96 \\
\hline 4 & $\xi$ & 98 & 98 & 98 & 100 \\
\hline 5 & $\overline{3}$ & 86 & 90 & 96 & 96 \\
\hline 6 & $\overline{3}$ & 84 & 96 & 98 & 100 \\
\hline 7 & $\vec{\tau}$ & 48 & 98 & 100 & 92 \\
\hline 8 & $\vec{\imath}$ & 66 & 86 & 98 & 96 \\
\hline 9 & ओ & 88 & 84 & 94 & 98 \\
\hline 10 & औ & 82 & 88 & 94 & 94 \\
\hline 11 & अं & 82 & 84 & 100 & 98 \\
\hline 12 & अ: & 56 & 62 & 98 & 98 \\
\hline 13 & $\overline{A C}$ & 50 & 62 & 76 & 92 \\
\hline
\end{tabular}

The performance comparison of the proposed approach with some existing approaches is presented in Table 5. 
Table 5: Summary of Offline Handwritten Hindi/ Devanagri Script Character Recognition Systems

\begin{tabular}{|c|c|c|c|c|c|c|}
\hline$\dot{\dot{z}}$ & 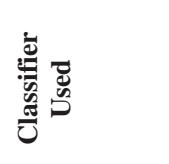 & 离 & 总 & 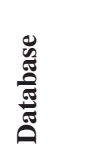 & 离 & 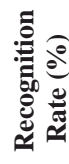 \\
\hline 1 & Fuzzy Sets & $\begin{array}{c}\text { Handwritten } \\
\text { Hindi } \\
\text { Characters } \\
\end{array}$ & $\begin{array}{c}\text { Modified } \\
\text { exponential } \\
\text { functions. }\end{array}$ & Own & $\begin{array}{c}\text { Hanmandlu } \\
\text { et al. } \\
{[8]} \\
\end{array}$ & 90.65 \\
\hline 2 & Fuzzy Sets & $\begin{array}{c}\text { Hindi } \\
\text { Numerals }\end{array}$ & $\begin{array}{c}\text { Normalize } \\
\text { distance } \\
\text { features, } \\
\text { Modified } \\
\text { exponential } \\
\text { functions }\end{array}$ & Own & $\begin{array}{c}\text { Hanmandlu } \\
\text { et al. } \\
\text { [9] }\end{array}$ & 96.0 \\
\hline 3 & Fuzzy Sets & $\begin{array}{c}\text { Isolated, } \\
\text { Devanagari } \\
\text { Characters } \\
\end{array}$ & $\begin{array}{l}\text { Structural } \\
\text { Features }\end{array}$ & Own & $\begin{array}{c}\text { Mukherji and } \\
\text { Rege } \\
{[11]} \\
\end{array}$ & 86.4 \\
\hline 4 & $\begin{array}{l}\text { Multi-layer } \\
\text { Perceptron }\end{array}$ & $\begin{array}{l}\text { Devanagari } \\
\text { Characters }\end{array}$ & $\begin{array}{l}\text { Three different } \\
\text { feature sets }\end{array}$ & Own & $\begin{array}{c}\text { Sandhya Arora } \\
\text { et al. } \\
\text { [12] }\end{array}$ & 92.16 \\
\hline 5 & $\begin{array}{c}\text { Backpropagation } \\
\text { Neural Network }\end{array}$ & $\begin{array}{c}\text { Handwritten } \\
\text { Hindi } \\
\text { Characters, } \\
\text { only Five } \\
\text { Starting } \\
\text { Consonants } \\
\end{array}$ & $\begin{array}{l}\text { Binary vector } \\
\text { of image }\end{array}$ & Own & $\begin{array}{c}\text { Gunjan Singh } \\
\text { et al. } \\
\text { [14] }\end{array}$ & 93.0 \\
\hline 6 & KNN & $\begin{array}{c}\text { Handwritten } \\
\text { Devanagari } \\
\text { Vowels }\end{array}$ & $\begin{array}{c}\text { Feature } \\
\text { extraction } \\
\text { using recursive } \\
\text { sub-division } \\
\text { techniques }\end{array}$ & $\begin{array}{c}\text { ISI } \\
\text { Kolkatta }\end{array}$ & $\begin{array}{c}\text { Rakesh Rathi } \\
\text { [15] }\end{array}$ & 96.14 \\
\hline 7 & SVM & $\begin{array}{c}\text { Hindi } \\
\text { Characters }\end{array}$ & $\begin{array}{c}\text { Diagonal } \\
\text { feature } \\
\text { extraction } \\
\end{array}$ & Own & $\begin{array}{c}\text { Sonika Dogra } \\
\text { et al. } \\
{[16]} \\
\end{array}$ & 93.06 \\
\hline 8 & $\begin{array}{l}\text { Backpropagation } \\
\text { Neural Network }\end{array}$ & $\begin{array}{c}\text { Isolated } \\
\text { Offline } \\
\text { Handwritten } \\
\text { Devanagari } \\
\text { Numerals }\end{array}$ & $\begin{array}{l}\text { Wavelets } \\
\text { features }\end{array}$ & $\begin{array}{c}\text { ISI } \\
\text { Kolkatta }\end{array}$ & $\begin{array}{c}\text { Gaurav Y. } \\
\text { Tawde } \\
\text { [17] }\end{array}$ & $60-70$ \\
\hline 9 & $\begin{array}{l}\text { Backpropagation } \\
\text { Neural Network }\end{array}$ & $\begin{array}{c}\text { Isolated } \\
\text { Offline } \\
\text { Handwritten } \\
\text { Hindi Script } \\
\text { Vowels }\end{array}$ & $\begin{array}{l}\text { Wave based } \\
\text { feature } \\
\text { extraction }\end{array}$ & Own & $\begin{array}{l}\text { Proposed } \\
\text { Method }\end{array}$ & 96.2 \\
\hline
\end{tabular}

\section{Conclusion}

The work aims at proposing an efficient approach for feature extraction to enhance the accuracy offline handwritten character recognition of Hindi characters. A novel technique 'TARANG', inspired by wave motion in a medium, for feature extraction is proposed to recognize offline handwritten Hindi 'SWARs' (vowels).

It is shown that when a feature vector obtained by using proposed wave based technique is used for training of Backpropagation Neural Network, a recognition rate as high as $95.7 \%$ is achieved. Also, when a feature set obtained by combining features with and without wave based approach is used for training neural network, a recognition rate as high as $96.2 \%$ is achieved, which is comparatively very high and also comparable with the accuracies some existing approaches. Further, better recognition rate is expected by dividing the image into smaller sized portions and then using the local wave features and global wave features to form the combined wave feature vector. A better accuracy is also expected by increasing the data set for training and testing the neural network. It is, therefore, concluded that the combined wave based feature vector is capable of producing even better results. The proposed approach is only implemented and tested for the samples of Hindi SWARs. In future, the proposed approach may be used with the entire character set of Hindi script as well as for other scripts using neural network or other classification scheme.

\section{References}

[1] Laurene Fausett, Fundamentals of Neural Networks, Architectures, Algorithms, and Applications, Addision Weslley Publishing Company, December, 1993.

[2] Birijesh K. Verma, "Handwritten Hindi Character Recognition Using Multilayer Perceptron and Radial Basis Function Neural Networks", IEEE, 0-7803-2768-3,1995, pp. 2111-2115.

[3] J.A. Anderson, Introduction to Neural Networks, MIT Press, 1995.

[4] Robert J. Schalkoff, Artificial Neural Networks, McGraw-Hill International Editions, Computer Science Series, 1997.

[5] L. S. Oliveira, N. Benahmed, R. Sabourn, F. Bortolozzi, and C. Y. Suen, "Feature Subset Selection Using Genetic Algorithms for Handwritten Digit Recognition", IEEE, 1530-1834, Vol. 37, 2001, pp. 362-369.

[6] Utpal Garain, Mangal P. Chakraborty, Dipankar Dasgupta, "Recognition of Handwritten Indic Script Using Clonal Selection Algorithm”, Springer Berlin/Heidelberg, 2006, pp. 256-266.

[7] Ranadhir Ghosh, Moumita Ghosh, "An Intelligent Offline Handwriting Recognition System Using Evolutionary Neural Learning Algorithm and Rule Based Over Segmented Data Points", Journal of Research and Practice in Information Technology, Vol. 37, No. 1, February 2005, pp. 73-87.

[8] M. Hanmandlu, O.V. Ramana Murthy, Vamsi Krishna Madasu "Fuzzy Model Based Recognition of Handwritten Hindi Characters", Digital Image Computing Techniques and Applications, IEEE, 0-7695-3067-2, 2007, pp. 454-461.

[9] M. Hanmandlu, A.V. Nath, A.C. Mishra, V.K. Madasu, "Fuzzy Model Based Recognition of Handwritten Hindi Numerals Using Bacterial Foraging", 6th IEEE/ACIS International Conference on Computer and Information Science (ICIS), IEEE, 0-7695-2841-4, 2007.

[10] U. Pal, T. Wakabayashi, F. Kimura, "Comparative Study of Devnagari Handwritten Character Recognition using Different Feature and Classifiers", IEEE, 10th International Conference on Document Analysis and Recognition, 2009, pp. 1111-1115.

[11] Prachi Mukherji, Priti P. Rege, "Shape Feature and Fuzzy Logic Based Offline Devnagari Handwritten Optical Character Recognition”, Journal of Pattern Recognition Research, 2009, pp. 52-68.

[12] Sandhya Arora, Debotosh Bhattacharjee, Mita Nasipuri, Dipak Kumar Basu, Mahantapas Kundu, "Multiple Classifier Combination for Offline Handwritten Devanagari Character Recognition", arxiv.org/pdf/1006.5913, June, 2010, pp. 01-06.

[13] Sheetal Dabra, Sunil Agrawal, Rama Krishna Challa, “A Novel Feature Set for Recognition of Similar Shaped Handwritten Hindi Characters Using Machine Learning", CCSEA, CS \& IT 02, 2011, pp. 25-35.

[14] Gunjan Singh, Sushma Lehri, "Recognition of Handwritten Hindi Characters using Backpropagation Neural Network", International Journal of Computer Science and Information Technologies, Vol. 3 (4), 2012, pp. 4892-4895.

[15] Rakesh Rathi, Ravi Krishan Pandey, Vikas Chaturvedi, Mahesh Jangid, "Offline Handwritten Devanagari Vowels Recognition using KNN Classifier", International Journal of Computer Applications, 09758887, Volume 49, No. 23, July 2012, pp. 11-16. 
[16] Sonika Dogra, Chandra Prakash, "Pahchaan: Hindi Handwritten Character Recognition System Based on SVM", International Journal on Computer Science and Engineering (IJCSE), 0975-3397 Vol. 4, No. 05, May 2012, pp. 718-722.

[17] Gaurav Y. Tawde, "Optical Character Recognition for Isolated Offline Handwritten Devanagari Numerals Using Wavelets", International Journal of Engineering Research and Applications, 2248-9622, Vol. 4, Issue 2 (Version 1), February 2014, pp.605-611.

[18] Kulkarni Sadanand A., Borde Prashant L., Manza Ramesh R., Yannawar Pravin L., "Offline MODI Character Recognition Using Complex Moments", Second International Symposium on Computer Vision and the Internet (VisionNet'15), Procedia Computer Science, 58516-523, 2001, pp-516-523.

Ajay Indian received the M.C.A. degree in 2001 from Dr. B.R Ambedkar University, Agra, India and M.Tech. (Computer Science \& Engineering) in 2010 from Gautam Buddha Technical University, Lucknow, India. Currently he is an Associate Professor at Department of Computer Science \& Applications, Invertis University, Bareilly, India and a research scholar at Department of Computer Science, Gurukula Kangri Vishwavidyalaya, Haridwar, India. His research interests include Image Processing, Artificial Neural Networks and Data Mining.

Karamjit Bhatia received M.Phil. degree in Computer Applications in 1988 from University of Roorkee (now IIT Roorkee), Roorkee, India and received Ph.D. (Computer Science) in 2001 from Gurukula Kangri Vishwavidyalaya, Haridwar, India. Currently he is a Professor at Department of Computer Science, Gurukula Kangri Vishwavidyalaya, Haridwar, India. His research interests include Distributed Systems, Image Processing and Mobile Ad-hoc Networks. He has published around 40 research papers in International Journals of repute and National/ International conference proceedings. Five Ph.D. degrees have been awarded under his supervision. 